\title{
Extended Computation: Wide Computationalism in Reverse
}

\author{
Paul Smart \\ University of Southampton \\ Southampton, United Kingdom \\ ps02v@ecs.soton.ac.uk
}

\author{
Wendy Hall \\ University of Southampton \\ Southampton, United Kingdom \\ wh@ecs.soton.ac.uk
}

\author{
Michael Boniface \\ University of Southampton \\ Southampton, United Kingdom \\ M.J.Boniface@soton.ac.uk
}

\begin{abstract}
Arguments for extended cognition and the extended mind are typically directed at human-centred forms of cognitive extensionforms of cognitive extension in which the cognitive/mental states and processes of a given human individual are subject to a form of extended or wide realization. The same is true of debates and discussions pertaining to the possibility of Web-extended minds and Internet-based forms of cognitive extension. In this case, the focus of attention concerns the extent to which the informational and technological elements of the online environment form part of the machinery of the (individual) human mind. In this paper, we direct attention to a somewhat different form of cognitive extension. In particular, we suggest that the Web allows human individuals to be incorporated into the computational/cognitive routines of online systems. These forms of computational/cognitive extension highlight the potential of the Web and Internet to support bidirectional forms of computational/cognitive incorporation. The analysis of such bidirectional forms of incorporation broadens the scope of philosophical debates in this area, with potentially important implications for our understanding of the foundational notions of extended cognition and the extended mind.
\end{abstract}

\section{CCS CONCEPTS}

-Human-centered computing; • Information systems $\rightarrow$ World Wide Web; • Social and professional topics $\rightarrow$ Computing / technology policy; • Computing methodologies $\rightarrow$ Artificial intelligence;

\section{KEYWORDS}

Extended Cognition, Extended Mind, Human Computation, Mechanisms, Socio-Technical Systems, World Wide Web, Distributed Cognition

\section{ACM Reference Format:}

Paul Smart, Wendy Hall, and Michael Boniface. 2021. Extended Computation: Wide Computationalism in Reverse. In 13th ACM Web Science Conference 2021 (WebSci '21 Companion), June 21-25, 2021, Virtual Event, United Kingdom. ACM, New York, NY, USA, 4 pages. https://doi.org/10.1145/3462741. 3466810

Recent work in the philosophy of mind and cognitive science has appealed to the idea that human mental states and processes may be subject to a form of wide realization-a form of realization in

Permission to make digital or hard copies of part or all of this work for personal or classroom use is granted without fee provided that copies are not made or distributed for profit or commercial advantage and that copies bear this notice and the full citation on the first page. Copyrights for third-party components of this work must be honored. For all other uses, contact the owner/author(s).

WebSci '21 Companion, June 21-25, 2021, Virtual Event, United Kingdom

(C) 2021 Copyright held by the owner/author(s)

ACM ISBN 978-1-4503-8525-1/21/06

https://doi.org/10.1145/3462741.3466810 which the mechanisms responsible for certain cognitive phenomena extend beyond the biological borders of the individual to which those phenomena are ascribed. This sort of idea lies at the heart of recent philosophical work pertaining to the notions of extended cognition and the extended mind [1, 2]. According to the hypothesis of extended cognition, for example, human cognitive states and processes are, on occasion, realized by mechanisms whose constituents include material elements that lie beyond the borders of the thing (the human individual) to which those cognitive states and processes are ascribed. In this case, the appeal to wide realization is made with regard to the sorts of phenomena that are the explanatory targets of contemporary cognitive science (e.g., extended memory, extended perception, extended problem-solving, and so on). This contrasts with the sorts of phenomena that animate discussions pertaining to the extended mind. In this case, the target phenomena include the mentalistic constructs invoked as part of folk psychological explanations of human behaviour. According to Clark and Chalmers [2], for example, extra-organismic resources, such as a paper-based notebook, can, on occasion, form part of the mechanistic supervenience base that sustains states of dispositional belief. In this case, a dispositional belief is ascribed to a particular human individual, but the physical mechanisms responsible for the manifest occurrence of that belief (i.e., the mechanisms responsible for the production of behaviour that warrants the ascription of the belief) are ones that include resources drawn from the individual's extra-organismic environment.

Over the past decade, there have been a number of attempts to apply these sorts of ideas to the Web and Internet. In terms of our own research, we have sought to examine the extent to which the informational and technological elements of the online environment might be incorporated into human cognitive processes $[10,11]$. A central feature of this work relates to the nature of human interactions and exchanges with the online environment. When it comes to claims about the Web-extended mind, for example, we have suggested that online information must be suitably poised to shape human thought and action in a manner that is consistent with the folk psychological role of belief states in the explanation and prediction of human behaviour. In more recent work, we have sought to apply this idea to the realm of extended knowledge. In particular, we have sought to develop Web-based applications that speak to the practical effort to engineer extended cognitive and epistemic systems [12].

One of the insights to emerge from this interdisciplinary effortwhich straddles both philosophy and Web science-relates to the status of both the online and offline environments as a source of building materials for the construction of extended computational and cognitive circuits. The best way of understanding this idea is 
via the notion of wide computationalism $[4,13]$. Wide computationalism seeks to sever the link between individualism and computational psychology by drawing attention to the wide [9] or extended [1] mechanisms that support cognitive-computational processes. This idea, it should be clear, is one that is easily accommodated by claims about the extended nature of human cognizing. Indeed, the only substantive difference between wide computationalism and extended cognition relates to the computational character of the cognitive phenomena (e.g., cognitive processes) that are realized by an extended nexus of material elements: The proponent of wide computationalism insists that human cognitive states and processes can be understood as bona fide computational phenomena, while the proponent of extended cognition remains largely uncommitted to this claim about the computational nature of the human cognitive system.

For present purposes, we will assume that human cognitive processes can be cast as computational processes and can thus be understood from a broadly computational perspective. The upshot is that when we consider the possibility of Web-based forms of cognitive extension, we are encouraged to think about our interactions and engagements with the Web and Internet (the online environment) in computational terms. That is to say, the processes that sustain episodes of (Web-based) extended cognizing will qualify as computational processes, and the informational and technological elements of the online environment will serve as the material elements of computational mechanisms that (in part) serve as the (wide) realization base for these processes. From the standpoint of wide computationalism, then, a case of Web-extended cognizing will qualify as a form of extended computation. Extended computation is, in short, the computational counterpart to extended cognition. It refers to a state-of-affairs in which a computational capacity is ascribed to a particular entity (in this case, a human individual), but the mechanisms that support the run-time realization of the processes reflecting the exercise of that capacity are ones that extend beyond the bounds of the entity to which the computational capacity is ascribed. (In the case of the Web and Internet, the processes reflecting the exercise of the computational capacity are ones that reach out to include the informational and technological elements of the online environment.)

At this point, we are in a position to establish a link between work in the philosophy of mind and computer science. In particular, we suggest that the notion of extended computation is one that is readily applicable to work on human computation systems $[5,8]$. A human computation system is a computational system that is partially constituted by the activities of one or more human individuals. That is to say, a human computation system is a system in which one or more human individuals are incorporated into the computational processing loops that would otherwise be implemented by a purely technological system (e.g., a conventional digital computer). ${ }^{1}$

What we see here is an effective reversal of the relationship between some extended entity (the entity that is subject to some form of extension) and the resources that are incorporated into the extended circuits that realize the manifest occurrence of the

\footnotetext{
${ }^{1}$ In fact, research in this area is not limited to the incorporation of human individuals: a variety of other animal species have been incorporated into computational processing loops [6].
}

capacities (and other dispositional properties) that are ascribed to that entity. From a philosophical and cognitive scientific standpoint, the focus of our attention is the human individual ( $q u a$ extended entity), and it is thus the elements of the extra-organismic environment (including the online environment) that we see as being the candidate elements for computational/cognitive incorporation. Relative to work on human computation systems, however, these roles are reversed. Now the focus of our attention is the online computational/technological system (qua extended entity) and it is the material elements of the offline (real-world) environment (e.g., human individuals) that are the material building blocks of extended computational circuits.

The upshot is that the appeal to extended computation highlights the bidirectional nature of the opportunities for computational/cognitive extension that are afforded by the Web and Internet. Relative to debates in the philosophy of mind and cognitive science, the human individual is the primary focus of our attention when it comes to a consideration of the potential for computational/cognitive extension, and it is thus the human individual that serves as what we might call the incorporating resource or extended entity-i.e., the entity that incorporates online resources into their computational/cognitive routines. This changes once we move beyond the browser interface and look at matters from the other side of the screen, so to speak. In this case, our attention shifts to consider the computational/cognitive capacities of the technological systems that inhabit the online world. Here, the human individual is not so much the incorporating resource as they are the incorporated resource-a resource that is incorporated into the computational/cognitive routines of an online system. What we have, then, is a distinction between two forms of computational/cognitive extension: When the human individual is subject to some form of extension, we have a case of human-centred computational/cognitive extension, while, from the technological standpoint, we have a case of machine-centred computational/cognitive extension.

As with human-centred forms of computational/cognitive extension, machine-centred forms of computational/cognitive extension raise a host of issues and concerns. Some of these concerns relate to the ethical implications of incorporating human individuals into extended processing loops. From a machine-centred perspective, human individuals emerge as the material fodder for the implementation of extended computational/cognitive circuits, and the role of the human individual is to service the computational/cognitive interests of the machine that incorporates them (just as our own human cognitive interests are served by the incorporation of material artefacts into our own cognitive processing routines). This raises a worry that is reminiscent of that expressed by social and political theorists of a bygone era. In respect of the Industrial Era, for example, Karl Marx remarked that "In handicrafts and manufacture, the worker makes use of a tool; in the factory, the machine makes use of him" [7, p. 548]. Our current philosophical preoccupation with human-centred forms of computational/cognitive extension is commensurate with Marx's views of human labour beyond the factory walls, for it is here that we see the incorporation of extra-organismic resources as a means of extending the reach of our human cognitive and epistemic horizons. The reversal of this relationship speaks to Marx's fears about the forms of exploitation and control that accompany the transition to life within the factory 
walls. For, in this case, the human individual is, according to Marx, relegated to the status of a mere component that serves the interests of the larger system (both mechanical and socio-economic) of which they are a part. In the context of debates about extended cognition and the extended mind, it is the elements of the external, extra-organismic world that become part of the machinery of the human mind. But, with the advent of the Web and Internet, we confront a somewhat more unnerving form of incorporation-a form of incorporation in which the human mind functions as a mere cog in a much larger computational/cognitive machine.

Setting aside the social and ethical concerns, we suggest that a consideration of human computation systems raises a number of issues that are central to philosophical debates about extended cognition and the extended mind. To help us see this, it is worth noting that from a purely mechanistic standpoint there is little to distinguish a case of human-centred computational/cognitive extension from a case of machine-centred computational/cognitive extension. Consider, for example, that the mechanisms underlying these two distinct forms of extension are ones that may be constituted by exactly the same components. From a human-centric perspective, a case of Web-based computational/cognitive extension will involve a human individual interacting with resources drawn from the online environment. But the same is true when we look at Web-based computational/cognitive extension from a machine-centric perspective. Once again, the mechanisms responsible for this particular form of extension will involve the interaction between some online resource (e.g., a computer) and some offline resource (e.g., a human individual). From a mechanistic standpoint, then, these two forms of Web-based computational/cognitive extension look to be indistinguishable. This is important, for it suggests that a mechanistic account of cognitive extension-one that appeals to the nature of the mechanisms responsible for the realization of extended computational/cognitive phenomena-is unlikely to be adequate (by itself) as a theoretical account of extended cognition and the extended mind.

A second issue relates to the distinction between distributed computation/cognition and extended computation/cognition. The term "distributed cognition" is typically invoked in the context of socio-technical systems--systems in which some form of cognitive processing occurs as the result of interactions and exchanges between a combination of social (human) and technological elements [see 3]. Given that this characterization is one that is readily applicable to a human computation system-at least when such systems are involved in the performance of a cognitive task-it is unclear how we ought to distinguish between the notions of distributed cognition and extended cognition. Are extended cognitive systems a particular kind of distributed cognitive system? Or are distributed cognitive systems a particular kind of extended cognitive system?

In addressing this issue, we suggest that we ought to direct attention to the entity that is deemed to be the subject of extended cognitive states and processes. A distinguishing feature of extended cognitive systems, we suggest, is that some dispositional property (e.g., a cognitive capacity, cognitive ability, or dispositional mental state) is ascribed to an entity, but the manifest occurrence of that property (e.g., the exercise of a cognitive ability) is one that involves the instantiation of a process whose (mechanistic) realization base includes elements that lie external to the entity to which the dispositional property is ascribed. This provides us with a way of distinguishing extended cognition from distributed cognition: In the case of distributed cognition, the dispositional properties are ascribed to the larger socio-technical system and the processes that reflect the manifest occurrence of those properties are ones that are realized by mechanisms whose constituents are confined to the borders of the thing (i.e., the socio-technical system) to which the dispositional properties are ascribed. This contrasts with the case of extended cognition, where the dispositional properties are ascribed to a single component of what (at run-time) amounts to a larger materially-hybrid system. Accordingly, we suggest that distributed cognition ought to be regarded as distinct from extended cognition: Distributed cognition is not a particular form of extended cognition, but neither is extended cognition a form of distributed cognition.

The same applies, we suggest, to the notions of distributed computation and extended computation. While it might seem appropriate to regard all human computation systems as engaged in distributed computation, we suggest that there is an important difference between distributed human computation systems and extended human computation systems. Distributed human computation systems are systems in which a computationally-relevant dispositional property is ascribed to a larger, socio-technically hybrid system. This contrasts with extended human computation systems, where the computationally-relevant dispositional properties are ascribed to the technological (in this case, online) component of the larger materially-hybrid (run-time) organization. From a machine-centric perspective, then, we will observe a case of extended computation whenever 1) we ascribe a computational capacity to an online technological system and 2) the exercise of this capacity is one that involves the instantiation of a computational process that includes resources (e.g., human individuals) that lie external to the technological system. The same will be true when we move from machine-centric forms of extended computation to machine-centric forms of extended cognition. In this case, the relevant capacity will be one that qualifies as a cognitive capacity, but this shift in the nature of the dispositional property will not affect the way we think about the extended status of that capacity. In both cases, the appeal to an extended capacity (regardless of whether this capacity is one that is cognitive or computational in nature) will be tied to the instantiation of a process whose realization base includes resources that lie external to the technological system to which the dispositional property is ascribed.

There is, no doubt, much more that could be written about these issues. For present purposes, however, we have sought to show that the philosophical preoccupation with Web-based forms of cognitive extension ought not to be limited to the idea that the online environment serves as a potential target for cognitive incorporation. By directing our attention to the notion of extended computation and the possibility for bidirectional forms of computational/cognitive incorporation, we are introduced to the possibility of machine-centric forms of computational/cognitive extension-forms of extension in which one or more human individuals are incorporated into the information processing loops of an online (technological) system. A consideration of such systems presents us with a bewildering array of social, ethical, and engineering concerns. From a philosophical perspective, however, the most important consideration is likely to be the way these peculiar forms of cognitive extension (which 
have emerged in the wake of the Web and Internet) introduce us to issues and concerns that might otherwise have been overlooked should our philosophical focus have remained firmly fixed on the traditional human targets of research into extended cognition and the extended mind.

\section{ACKNOWLEDGMENTS}

This work is supported by the UK EPSRC as part of the PETRAS National Centre of Excellence for IoT Systems Cybersecurity under Grant Number EP/S035362/1 (https://petras-iot.org//).

\section{REFERENCES}

[1] Andy Clark. 2008. Supersizing the Mind: Embodiment, Action, and Cognitive Extension. Oxford University Press, New York, New York, USA.

[2] Andy Clark and David Chalmers. 1998. The Extended Mind. Analysis 58, 1 (1998), 7-19.

[3] Edwin Hutchins. 1995. Cognition in the Wild. MIT Press, Cambridge, Massachusetts, USA.
[4] Luke Kersten. 2017. A Mechanistic Account of Wide Computationalism. Review of Philosophy and Psychology 8, 3 (2017), 501-517.

[5] Edith Law and Luis von Ahn. 2011. Human Computation. Synthesis Lectures on Artificial Intelligence and Machine Learning 5, 3 (2011), 1-121.

[6] Richard M Levenson, Elizabeth A Krupinski, Victor M Navarro, and Edward A Wasserman. 2015. Pigeons (Columba livia) as trainable observers of pathology and radiology breast cancer images. PLoS One 10, 11 (2015), e0141357.

[7] Karl Marx. 1976. Capital: A Critique of Political Economy. Vol. 1. Penguin Books Ltd, Harmondsworth, Middlesex, England.

[8] Pietro Michelucci (Ed.). 2013. Handbook of Human Computation. Springer, New York, New York, USA.

[9] Marcin Miłkowski, Robert Clowes, Zuzanna Rucińska, Aleksandra Przegalińska, Tadeusz Zawidzki, Joel Krueger, Adam Gies, Marek McGann, Łukasz Afeltowicz, Witold Wachowski, Fredrik Stjernberg, Victor Loughlin, and Mateusz Hohol. 2018. From wide cognition to mechanisms. Frontiers in Psychology 9, Article 2393 (2018), 1-17.

[10] Paul R Smart. 2012. The Web-Extended Mind. Metaphilosophy 43, 4 (2012), 426-445.

[11] Paul R Smart. 2017. Extended Cognition and the Internet: A Review of Current Issues and Controversies. Philosophy \& Technology 30, 3 (2017), 357-390.

[12] Paul R Smart. submitted. Shedding Light on the Extended Mind: HoloLens, Holograms, and Internet-Extended Knowledge. Frontiers in Psychology (submitted).

[13] R. A. Wilson. 1994. Wide computationalism. Mind 103, 411 (1994), 351-372. 